\title{
Secondary iron mineralization upon Fe(II) sorption to montmorillonite
}

\author{
RichARD N COLLINS*, XIN WANG AND ADELE M \\ JONES \\ School of Civil and Environmental Engineering, University \\ of New South Wales, Sydney, NSW 2052, Australia. \\ (*correspondence: richard.collins@unsw.edu.au)
}

The sorption of $\mathrm{Fe}$ (II) to smectite clay minerals is a ubiquitous reaction expected to occur in both natural and engineered environments. These systems encompass redox active marine, coastal and terrestrial systems as well as anthropogenic clay barriers typically used to isolate waste from the surrounding (sub-surface) natural environment. Upon Fe(II) sorption to montmorillonite a range of secondary reactions can take place including electron transfer, clay mineral dissolution and the precipitation of secondary iron minerals - all of which can potentially affect the fate of associated contaminants.

I will present the results of laboratory studies that we have undertaken to determine the stability fields of the major secondary iron minerals that form when Fe(II) sorbs to montmorillonite (consisting of $\sim 2 \%$ structural $\mathrm{Fe}(\mathrm{III})$ ), primarily as a function of $\mathrm{pH}$ and, as a result, the degree of $\mathrm{Fe}(\mathrm{II})$ sorption. We have observed that low Fe(II) surface loadings leads to interfacial electron transfer between sorbed $\mathrm{Fe}(\mathrm{II})$ to clay structural $\mathrm{Fe}$ (III) with the concurrent precipitation of ferrihydrite. Despite the abundance of Fe(II) present, we do not observe the $\mathrm{Fe}(\mathrm{II})$-catalysed transformation of ferrihydrite to either lepidocrocite or goethite.

At higher Fe(II) surface loadings, between $\mathrm{pH} 7$ to 7.5 , the precipitation of $\mathrm{Fe}$ (II) becomes a significant mechanism and the dominant secondary iron mineral that forms is green rust. This mineral forms regardless of whether the major anion present in the medium is either chloride, sulfate or carbonate and, thus, provides insight into the possible relevance of this secondary iron mineral formation pathway across a range of diverse aquatic environments.

I will conclude on recounting preliminary experiments examining the ability of the different forms of $\mathrm{Fe}(\mathrm{II})$ in these systems to reduce nitrate $\left(\mathrm{NO}_{3}\right)$ - a notoriously difficult, though thermodynamically favorable, abiotic electron transfer process. 